\title{
Consensus Problem for Linear Time-Invariant Systems with Time-Delay
}

\author{
V. Fragoso-Rubio ${ }^{\mathbb{D}},{ }^{1}$ M. Velasco-Villa ${ }^{\mathbb{D}},{ }^{1}$ M. A. Vallejo-Alarcón $\mathbb{D}$, ${ }^{1}$ \\ J. A. Vásquez-Santacruz $\mathbb{D}^{2}{ }^{2}$ and M. A. Hernández-Pérez ${ }^{3}$ \\ ${ }^{1}$ Departamento de Ingeniería Eléctrica, Sección de Mecatrónica, CINVESTAV-IPN, Av. IPN, No. 2508, \\ Col. San Pedro Zacatenco, 07300 Ciudad de México, Mexico \\ ${ }^{2}$ Universidad Veracruzana, Facultad de Ingeniería, Juan Pablo II $s / n$, Boca del Río, Veracruz, Mexico \\ ${ }^{3}$ Universidad Veracruzana, Instituto de Ingeniería, Juan Pablo II s/n, Boca del Río, Veracruz, Mexico
}

Correspondence should be addressed to M. Velasco-Villa; velasco@cinvestav.mx

Received 19 March 2019; Revised 11 June 2019; Accepted 20 June 2019; Published 7 July 2019

Academic Editor: Francesco Conte

Copyright (C) 2019 V. Fragoso-Rubio et al. This is an open access article distributed under the Creative Commons Attribution License, which permits unrestricted use, distribution, and reproduction in any medium, provided the original work is properly cited.

\begin{abstract}
This work deals with the consensus problem of networks of agents with linear time-invariant dynamics and input time-delay. A predictor-observer scheme that estimates the future value of the system state is considered. The partitioned nature of the predictor allows dealing with larger time-delays than those reported in the literature. The estimated future state of the system is later used in the consensus protocol with the aim of compensating the system input delay. The effectiveness of the solution is shown by means of numerical evaluations.
\end{abstract}

\section{Introduction}

Interest in multiagent systems is due to the multiple possible applications of this kind of systems such as the formation problem of Unmanned Aerial Vehicles (UAV), groups of satellites, formation control vehicles, distributed sensors, automatic vehicular control, and communication networks; for instance, see [1-3]. Consensus problem is referred to as the control and coordination of a set of agents within a communication topology. In the study of consensus problem for multiagent systems, factors as communication topology, time-delays, and dynamic model become relevant [4-7]. The importance of the Laplacian of the network in the solution of the consensus problem has been studied in $[1,2,8]$. The consensus problem becomes a more challenging task when the communication topology among agents presents time-delays. Several solutions have been reported considering different system conditions as fixed time-delay and topologies [9], multiple time-delay cases [10, 11], systems with topologies that change over time [12], and also systems within a discrete-time context [10]. Consensus problem for systems with single integrator dynamics has been widely studied $[4,5,13]$; solutions for agents with double integrator dynamics are presented in works such as $[9,13]$. High-order linear systems have been dealt with in [7], where consensus problem is converted in the simultaneous stabilization of multiple subsystems and sufficient conditions for consensus and consensualization independent of the number of agents in the systems are presented; this work is extended in [14] by considering variable time-delays. Linear time-invariant (LTI) systems with variable topologies and multiple time-delays are considered in [15] and necessary and sufficient conditions for consensus are given. A PD-like consensus scheme that transforms a system with constant or variable delays into a neutral type system is presented in [16]. Prediction-based schemes have been proposed for systems without time-delays to estimate the current state of the agents when only relative measurements are available. For systems with time-delay the consensus problem of single integrator agents with input and output time-delay is studied in [17] where a state predictor is used to compensate the time-delay, while consensus-based formation of agents with double integrator dynamics [18] and heterogeneous systems composed of agents with single and double integrators [19] are studied and the input time-delay 
is compensated by means of a state predictor. The consensus problem of high-order multiagent systems with input and output communication delays is solved in [20] by means of a truncated predictor-based scheme. The truncated predictor approach is extended in [21] to compensate the input timedelay of nonlinear systems in order to achieve consensus, while a nonlinear adaptive observer estimates the states of the agents that are used in the adaptive control scheme that is proposed in [22] to achieve leader-following consensus for uncertain nonlinear multiagent systems.

The control problem of systems with time-delays has also been of the interest of the scientific community. To overcome this difficulty, many solutions have been presented. A first approach was the Smith Predictor [23], composed by a control designed without considering the system time-delay and a prediction of the output that compensates the delay. Several modifications of the scheme were later presented to overcome its shortcomings and to expand the types of systems where it is applicable; see $[24,25]$ being some of these works. The assignment of a finite spectrum of the closedloop system (Finite Spectrum Assignment) is the goal of the approach presented in [26]. The reduction strategy in [27] is motivated by a similar idea. However, in these solutions, the dimension of the feedback law is infinite, which implies that the implementation of this method must be realized by means of a numerical approximation. Safe numerical implementations are presented in $[28,29]$.

The prediction approach took a step further in [30] where the state prediction is attained for linear systems with both input and state delays. Nonetheless, these chained observers are composed by distributed terms, in which implementation requires a numerical approximation. It is in the work of Najafi et al. [31] where a group of chained predictors with finite spectrum is proposed to compensate the input delay of linear MIMO systems. The approach assumes that the whole system state can be measured and guarantees asymptotic stability of the closed-loop system and prediction error. The stability proof of the closed-loop system results in a method to calculate the gain matrices of the predictor and the control by means of Linear Matrix Inequalities (LMI). The work is extended in [32] where robust $H_{\infty}$ control is used to minimize the input disturbance effect on the prediction error for linear systems with input time-delay. The idea of a sequence of predictors has been applied to linear systems with time-varying input [33] and time-varying output [34] time-delays and to time-varying systems with time-varying delays [35]. In [36] the state of a nonlinear system with delayed output is reconstructed by means of a chained set of observers. The main advantage of the chained predictors resides on their ability to deal with larger time-delays.

Based on the idea of the nested predictor [31, 34], the present paper deals with the consensus problem of LTI systems with constant time-delay by proposing a modification of the predictor-observer scheme presented in [37] that estimates the state of the system forecasted $\tau$ units of time into the future. The predictor assumes that only a part of the state space can be measured and the definition of the subpredictor errors allows proving that its convergence implies the convergence of the predicted state to the future system state. The estimated future state is later on used by the consensus algorithm, to compensate the input delay of the system. It is important to note that since there is a timedelay in the input of the systems, by using the forecasted estimated state, the closed-loop system will be in terms of the current state which results in the compensation of the input time-delay. The consensus protocol presented here is an adaptation of the one proposed in [7] for LTI systems and initially adapted for the input delay case in [38]. The modification to the protocol that we propose consists of using the predicted future state instead of the current and delayed states, which allows systems with larger delays to achieve consensus, as it will be shown in the numerical evaluations.

The work is organized as follows, in Section 2 a brief revision of the graph theory and the properties of the Laplacian matrix is presented, Section 3 presents the statement of the problem, and then the predictor-observer scheme that allows estimating the future state of the system is developed in Section 4. The consensus algorithm is given in Section 5 and the results of the numerical evaluations can be found in Section 6. The work ends with some conclusions in Section 7.

\section{Graph Theory and Laplacian Matrix}

Graph theory is the mathematical tool used to model the information exchange among agents [39]. This section presents some basic concepts of graph theory and properties of the Laplacian matrix that are of the interest of this work.

Let $\mathscr{G}=\{\mathcal{N}, \mathscr{E}\}$ be an ordered graph where $\mathcal{N}$ is the nonempty node set, $\mathcal{N}=\{1, \ldots, n\}$, and $\mathscr{E} \subset \mathcal{N} \times \mathcal{N}$ is the edge set. An edge in $\mathscr{G}$ is given by $e_{i j}=(i, j)$.

A directed graph is a pair $(\mathcal{N}, \mathscr{E})$ where the edge $e_{i j} \in \mathscr{E}$ indicates that agent $j$ can obtain information from agent $i$, but not necessarily in the other way. The edge $(i, j)$ has a node $i$ that is called the parent node and a node $j$ called child node. A directed path is an edge sequence in a directed graph of the form $\left(i_{1}, i_{2}\right),\left(i_{2}, i_{3}\right), \ldots$. A directed tree is a directed path with every node having exactly one parent, except for a node called root node that has no parent node and has directed paths to every other node. A subgraph $\left(V^{s}, \mathscr{E}^{s}\right)$ of $(V, \mathscr{E})$ is such that $V^{s} \subset V$ and $\mathscr{E}^{s} \subset \mathscr{E} \cap\left(V^{s} \times V^{s}\right)$. A directed spanning tree $\left(V^{s}, \mathscr{E}^{s}\right)$ of the directed graph $(V, \mathscr{E})$ is a subgraph with a directed path where $V^{s}=V$.

The adjacency matrix $A=\left[a_{i j}\right] \in \mathfrak{R}^{n \times n}$ of a directed graph with the set node $\mathcal{N}=\{1, \ldots, n\}$ is such that the weight $a_{i j}$ is positive if $(i, j) \in \mathscr{E}$ and $a_{i j}=0$ if $(i, j) \notin \mathscr{E}$. When weights are not relevant, $a_{i j}=1$ if $(i, j) \in \mathscr{E}$.

2.1. Laplacian Matrix. The Laplacian matrix $\mathscr{L}=\left[l_{i j}\right] \in \mathfrak{R}^{n \times n}$ of a directed graph is given by $l_{i i}=\sum_{j=1, j \neq i}^{n} a_{i j}$ and $l_{i j}=-a_{i j}$ for every $i \neq j$. Some remarkable properties of this matrix are considered.

(i) $l_{i j} \leq 0, i \neq j$.

(ii) The sum of the elements of every row equals zero. $\sum_{j=1}^{n} l_{i j}=0, i=1, \ldots, n$.

(iii) For directed and undirected graphs, $\lambda_{L, 1}=0$ is an eigenvalue of $\mathscr{L}$ due to the fact that the sum of all 
the elements of a row equals zero. There also exists an eigenvector $1_{n}=[1, \ldots, 1]^{T}$, with $n \times 1$ dimensions corresponding to the zero eigenvalue.

(iv) All of the eigenvalues $\lambda_{L, i}$ of $\mathscr{L}$, different from zero, are positive (for directed graphs) or have positive real parts (for undirected graphs). Therefore, the real part of every eigenvalue of $-\mathscr{L}$ is negative.

\section{Problem Statement}

This work deals with the consensus problem for a set of agents with input time-delay. The dynamics of the agents is modeled as linear time-invariant systems with input time-delay and fixed communication topology. The graph that models the communication is considered to be either directed with a spanning tree or undirected and connected, which implies that one of the eigenvalues of the Laplacian matrix equals zero [40].

Each agent is considered to be a $p$-dimensional MultipleInput-Multiple-Output (MIMO) linear system with the same input time-delay and dynamics given by

$$
\begin{aligned}
& \dot{x}_{i}(t)=A_{i} x_{i}(t)+B_{i} u_{i}\left(t-\tau_{i}\right) \\
& y_{i}(t)=C_{i} x_{i}(t),
\end{aligned}
$$

for $i=1,2, \ldots, n$, where $x_{i}=\left[x_{i 1}, \ldots, x_{i p}\right]^{T} \in \mathfrak{R}^{p}$ represents the state of the $i$-th agent, $u_{i} \in \mathfrak{R}^{q}$ is the input control, $\tau_{i}>0$ is a known constant input time-delay, $y_{i} \in \mathfrak{R}^{r}$ is the output signal, and $A_{i} \in \mathfrak{R}^{p \times p}, B_{i} \in \mathfrak{R}^{p \times q}$, and $C_{i} \in \mathfrak{R}^{r \times p}$. It is important to note that every parameter is associated with the $i$-th agent.

Consensus Problem. A system of $n$ agents is said to achieve consensus if for any initial condition $x_{i}(0) ; x_{i}(t) \longrightarrow x_{j}(t)$ as $t \longrightarrow \infty$, for all $i, j=1, \ldots, n[1]$.

To solve the consensus problem for multiagent systems a protocol is presented in [7], for agents without communication delay with dynamics given by

$$
\dot{x}_{i}(t)=A_{i} x_{i}(t)+B_{i} u_{i}(t)
$$

The consensus protocol is given by

$$
u_{i}(t)=K_{1} x_{i}(t)+K_{2} \sum_{j=1}^{n} a_{i j}\left(x_{j}(t)-x_{i}(t)\right)
$$

for $i, j=1, \ldots, n$, where $a_{i j}$ are the elements of the adjacent matrix and $K_{1}$ and $K_{2}$ are constant gain matrices. The authors present necessary and sufficient conditions for consensus and consensualization.

\section{Predictor-Observer Scheme}

To compensate the time-delay present in the dynamics of the agents, a predictor-observer scheme is proposed. The objective is to estimate the future state of the agents $\tau_{i}$ units of time ahead, to later design a consensus scheme based on the predicted state. It is important to note that the use of predicted future states on time-delayed systems avoids the numerical implementation problems present in solutions with infinite spectrum; even more, the partitioned nature of the scheme proposed by this work allows compensating large time-delays. The design of the prediction-observer scheme is done under the following assumptions.

Assumption 1. Signals $y(t)$ and $u(t)$ are available for measurement.

Assumption 2. For system (1), the pair $\left(A_{i}, B_{i}\right)$ is controllable and $\left(A_{i}, C_{i}\right)$ is observable.

Assumption 3. Consider that for the time delay $\tau_{i}$ in (2) there exists an integer $m_{i}>0$ such that $\bar{\tau}_{i}=\tau_{i} / m_{i}$.

Remark 4. Notice that Assumption 3 is just a technical requirement in order to avoid the consideration of irrational values for the time-delay $\tau_{i}$ that could yield an approximate prediction.

For the $i$-th agent, a set of advanced variables can be defined as

$$
\begin{gathered}
w_{i_{1}}(t)=x_{i}\left(t+\bar{\tau}_{i}\right) \\
w_{i_{2}}(t)=w_{i_{1}}\left(t+\bar{\tau}_{i}\right)=x_{i}\left(t+2 \bar{\tau}_{i}\right) \\
\vdots \\
w_{i_{m}}(t)=w_{i_{m-1}}\left(t+\bar{\tau}_{i}\right)=x_{i}\left(t+m \bar{\tau}_{i}\right)=x_{i}\left(t+\tau_{i}\right),
\end{gathered}
$$

where $w_{i_{f}}=\left[w_{i 1_{f}}, w_{i 2_{f}}, \ldots, w_{i p_{f}}\right]^{T}$ for $f=1, \ldots, m$ refers to the $p$-dimensional vector state. Taking the time derivative of (4), it is obtained that

$$
\dot{w}_{i_{f}}=A_{i} w_{i_{f}}+B_{i} u\left(t-(m-f) \bar{\tau}_{i}\right),
$$

for $f=1, \ldots, m$. In particular, for $f=m$,

$$
\dot{w}_{i_{m}}=A_{i} w_{i_{m}}+B_{i} u(t)
$$

and a free-delay dynamic is obtained.

Notice that system (6) represents the evolution of system (1) forecasted $\tau_{i}$ units of time without input-delay.

For system (6), the following predictor-observer is proposed:

$$
\dot{\widehat{w}}_{i_{f}}=A_{i} \widehat{w}_{i_{f}}+B_{i} u_{i}\left(t-(m-f) \bar{\tau}_{i}\right)+L_{i} C_{i} e_{w i_{f}}\left(t-\bar{\tau}_{i}\right)
$$

for $f=1, \ldots, m$ where $L_{i} \in \mathfrak{R}^{p \times r}$ is a gain matrix and the error signal

$$
e_{w i_{f}}(t)=\left[e_{w i 1_{f}}(t), \ldots, e_{w i p_{f}}(t)\right]^{T}
$$

takes the form

$$
\begin{aligned}
& e_{w i_{1}}(t)=x_{i}\left(t+\bar{\tau}_{i}\right)-\widehat{w}_{i_{1}}(t) ; \\
& e_{w i_{f}}(t)=\widehat{w}_{i_{f-1}}\left(t+\bar{\tau}_{i}\right)-\widehat{w}_{i_{f}}(t) ; \text { for } f=2,3, \ldots, m .
\end{aligned}
$$


The error dynamics (9) is given by

$$
\begin{aligned}
& \dot{e}_{w i_{1}}(t)=\dot{x}_{i}\left(t+\bar{\tau}_{i}\right)-\dot{\widehat{w}}_{i_{1}}(t) ; \\
& \dot{e}_{w i_{f}}(t)=\dot{\hat{w}}_{i_{f-1}}\left(t+\bar{\tau}_{i}\right)-\dot{\hat{w}}_{i_{f}}(t) ;
\end{aligned}
$$

$$
\text { for } f=2,3, \ldots, m \text {, }
$$

resulting in

$$
\begin{aligned}
\dot{e}_{w i_{1}}(t)= & A_{i} e_{w i_{1}}(t)-L_{i} C_{i} e_{w i_{1}}\left(t-\bar{\tau}_{i}\right) ; \\
\dot{e}_{w i_{f}}(t)= & A_{i} e_{w i_{f}}(t)+L_{i} C_{i} e_{w i_{f-1}}(t) \\
& -L_{i} C_{i} e_{w i_{f}}\left(t-\bar{\tau}_{i}\right)
\end{aligned}
$$

with $f=2,3, \ldots, m$. The vectorial form of (11) is the timedelay error system given by

$$
\dot{e}_{w i}(t)=A_{0}^{*} e_{w i}(t)+A_{1}^{*} e_{w i}\left(t-\bar{\tau}_{i}\right),
$$

with $e_{w i}=\left[e_{w i_{1}}, \ldots, e_{w i_{m}}\right]^{T}$ for $i=1, \ldots, n$ and

$$
\begin{aligned}
A_{0}^{*} & =\left[\begin{array}{ccccc}
A_{i} & \mathbf{0} & \cdots & \mathbf{0} & \mathbf{0} \\
L_{i} C_{i} & A_{i} & \cdots & \mathbf{0} & \mathbf{0} \\
\vdots & \vdots & \ddots & \vdots & \vdots \\
\mathbf{0} & \mathbf{0} & \cdots & L_{i} C_{i} & A_{i}
\end{array}\right], \\
A_{1}^{*} & =\left[\begin{array}{ccc}
-L_{i} C_{i} & \cdots & \mathbf{0} \\
\vdots & \ddots & \vdots \\
\mathbf{0} & \cdots & -L_{i} C_{i}
\end{array}\right],
\end{aligned}
$$

where $\mathbf{0} \in \mathfrak{R}^{p \times p}$ is a matrix with all inputs equal to zero.

4.1. Related Predictor Scheme. The predictor given by (7) is equivalent to the sequential subpredictors strategy proposed by [31] that is recalled in the following.

Consider system (1) and assume that all the state $x(t)$ is available for measurement; this is $C_{i}=I$;

$$
\dot{x}(t)=A x(t)+B u(t-\tau),
$$

and there exists $m \in \mathscr{Z}^{+}$such that

$$
\bar{\tau}=\frac{\tau}{m},
$$

which allows proposing the sequence of subpredictors given by

$$
\begin{gathered}
\dot{\bar{x}}_{1}(t)=A \bar{x}_{1}(t)+L_{1}\left(\bar{x}_{1}(t-\bar{\tau})-\bar{x}_{2}(t)\right)+B u(t) \\
\vdots \\
\dot{\bar{x}}_{i}(t)=A \bar{x}_{i}(t)+L_{i}\left(\bar{x}_{i}(t-\bar{\tau})-\bar{x}_{i+1}(t)\right) \\
\quad+B u(t-(i-1) \bar{\tau})
\end{gathered}
$$

$$
\begin{aligned}
& \dot{\bar{x}}_{m}(t)=A \bar{x}_{m}(t)+L_{m}\left(\bar{x}_{m}(t-\bar{\tau})-\bar{x}_{m+1}(t)\right) \\
& \quad+B u(t-(m-1) \bar{\tau})
\end{aligned}
$$

with $\bar{x}_{i} \in \mathfrak{R}^{n}, i=1, \ldots m$. The prediction errors for each subpredictor are defined as

$$
\begin{aligned}
e_{i}(t) & =\bar{x}_{i}(t-(m-i-1) \bar{\tau})-\bar{x}_{i+1}(t-(m-1) \bar{\tau}) \\
e_{m}(t) & =\bar{x}_{m}(t-\bar{\tau})-x(t),
\end{aligned}
$$

for $i=1, \ldots m-1$ and the error dynamics given by

$$
\begin{aligned}
\dot{e}_{i}(t) & =A e_{i}(t)+L_{i} e_{i}(t-\bar{\tau})-L_{i+1} e_{i+1}(t-\bar{\tau}) \\
\dot{e}_{m}(t) & =A e_{m}(t)+L_{m} e_{m}(t-\bar{\tau}),
\end{aligned}
$$

for $i=1, \ldots, m-1$. Asymptotic convergence for the error dynamics is guaranteed by means of an LMI method that considers the closed-loop system that allows calculating the subpredictors and controlling gain matrices.

The main difference between both schemes is that authors in [31] consider systems where it is possible to read the whole state and the predictor-observer (7) presented here includes the cases when only a partial measurement is available. The relation between both schemes can be found considering the following coordinate change:

$$
\widehat{w}_{1}(t)=\bar{x}_{m}(t)
$$

$$
\widehat{w}_{m}(t)=\bar{x}_{1}(t),
$$

and for the error signals

$$
e_{w_{i}}(t)=-e_{m+1-i}(t+(m+1-i) \bar{\tau}),
$$

for $i=1, \ldots m$, to subpredictors (16) and prediction errors (17) and assuming $C=I$ in system (1) results in the chain of predictors (7) and error signals (12).

Remark 5. It is important to note that the predictor (16) cannot be used to estimate the future state of system (1) when $C_{i} \neq I$.

4.2. Convergence Analysis of the Predictor-Observer. The convergence to zero of the injection errors (9) is assured by showing the stability of the error dynamics (12). 
Lemma 6. Let the predictor-observer (7) satisfy Assumptions 1,2 , and 3. Suppose that there exist positive gain matrices $L_{i}$ for $i=1,2, \ldots, n$ such that the matrix $\left(A_{i}-L_{i} C_{i}\right)$ is Hurwitz; then there exists a sufficiently large $m_{i}$ such that the error signals (9) exponentially converge to zero.

Proof. The characteristic equation of (12) is given by

$$
p(s)=\operatorname{det}\left(s I_{m p}-A_{0}^{*}-A_{1}^{*} e^{-s \bar{\tau}_{i}}\right)=0 .
$$

It is possible to show that (21) can be rewritten as

$$
p(s)=\prod_{f=1}^{m}\left(I_{n} s-A_{i}+L_{i} C_{i} e^{-s \tau_{i}}\right)=0 .
$$

It is clear that every factor of (22) represents the Laplace transform of a system of the form

$$
\dot{e}_{w i, f}(t)=A_{i} e_{w i, f}(t)-L_{i} C_{i} e_{w i, f}\left(t-\bar{\tau}_{i}\right) .
$$

Since the parameters of $L_{i}$ are free, from the observability assumption of the pair $\left(A_{i} C_{i}\right)$, it is always possible to locate the eigenvalues of the matrix $A_{i}-L_{i} C_{i}$ in the left half complex plane. The Hurwitz property of matrix $A_{i}-L_{i} C_{i}$ assures the stability of the differential difference equation (23) for a sufficiently small time delay $\bar{\tau}_{i}$ [41]. The maximum time delay $\tau_{i}^{*}$ for which system (23) will be asymptotically stable depends on the choice of the observer gain $L_{i}$ and its computation is not an easy task since its complexity rises as the dimension of the system increases. Thus, given a gain $L_{i}$, such that $A_{i}-L_{i} C_{i}$ is a Hurwitz matrix, it is always possible to asymptotically stabilize the prediction error (23) for a constant time delay $\tau_{i}<\tau_{i}^{*}$. This fact can be verified, for instance, by considering [42] or by reviewing the pole assignment procedure given in [41]. Based on the above arguments, it will be assumed, without loss of generality, that there exist $L_{i}$ and $\tau_{i}^{*}$ such that system (23) is asymptotically stable for $0 \leq \tau_{i}<\tau_{i}^{*}$. It should also be noticed that the stability of (23) is not only asymptotic but also exponential [43]. Furthermore, if matrix $L_{i}$ is chosen according to the rule given in Theorem 3.1 in [34], this is

$$
L=e^{\bar{A}_{o} \tau^{*}}
$$

with

$$
\bar{A}_{o}=A-\bar{L} C,
$$

where $\bar{L}$ is such that all the real parts of the eigenvalues of $\bar{A}_{o}$ are smaller than some negative real constant $\alpha$; it is possible to guarantee the exponential convergence of the prediction errors $e_{w i f}(t)$ to zero, which results in the convergence of the estimated future state $\widehat{w}_{i m}(t)$ to the real future value $x\left(t+\tau_{i}\right)$.

Remark 7. It should be pointed out that a predictor of the form (23) with $m=1$ designed for a system with time delay at the output was considered in [25]; also, for the case $m=1$, in [44] a predictor for a nonlinear input delay system is designed. As mentioned before, the partitioned predictor presented in [31] is similar to the one presented in this section, considering $C=I$.
Remark 8. Notice that in the case of $\bar{\tau}_{i}=0$, the error dynamics (23) takes the form

$$
\dot{e}_{w i, f}(t)=\left(A_{i}-L_{i} C_{i}\right) e_{w i, f}(t)
$$

where the stability is determined by the eigenvalues of the matrix $A_{i}-L_{i} C_{i}$ that, by the observability assumption, can always be stabilizable.

Remark 9. It should be noticed that $\tau_{i}$ is given by $\tau_{i}=$ $m \bar{\tau}_{i}$; therefore the larger number of subdelays considered by scheme (7) is, the larger delay $\tau_{i}$ can be compensated, with the drawback of an increasing dimension of the proposed predictor-observer.

The following result allows obtaining the estimated values $\widehat{x}_{i}\left(t+f \bar{\tau}_{i}\right)$ from the future real values $x_{i}\left(t+f \bar{\tau}_{i}\right)$ in (4).

Lemma 10. Suppose for system (1) that Assumptions 1, 2, and 3 are satisfied. For every $\tau_{i}>0$, there always exists a predictorobserver (7) such that the estimated states $\widehat{w}_{i_{f}}(t)$ exponentially converge to the future values $x_{i}\left(t+f \bar{\tau}_{i}\right)$ for $f=1, \ldots, m$.

Proof. From the definition of error (9) it is possible to show that

$$
\widehat{w}_{i_{f}}(t)=x\left(t+f \bar{\tau}_{i}\right)-\sum_{k=1}^{f} e_{w i_{k}}\left(t+(f-k) \bar{\tau}_{i}\right)
$$

for $k=1, \ldots, f$. Then, by Lemma 6 , it is possible to conclude that the estimated states $\widehat{w}_{i_{f}}(t)$ exponentially converge to the corresponding real values.

Remark 11. Note that if $m \longrightarrow \infty$, then $\bar{\tau}_{i} \longrightarrow 0$, relaxing the convergence conditions of the estimation error. This implies that it is always possible to stabilize system (12) choosing the integer $m$ large enough.

\section{Consensus Problem}

In this section, the consensus protocol for LTI systems in time-delay is presented. The set of agents has a fixed communication topology that contains a directed spanning tree. The dynamics of each agent is given by (1). This protocol considers the solution presented in [7] that is adapted to be used with the future state values estimated in the previous section. By using the predicted states instead of the current one, it is possible to deal with systems with large time-delays.

Consider a set of $n$ agents of the form (1), where for the sake of easy of presentation it is assumed that $\tau_{i}=\tau$ for $i=$ $1, \ldots, n$; that is,

$$
\dot{x}_{i}(t)=A x_{i}(t)+B u_{i}(t-\tau) .
$$
by

The vectorial form of the complete set of agents is given

$$
\dot{x}(t)=\left(I_{n} \otimes A\right) x(t)+\left(I_{n} \otimes B\right) u(t-\tau)
$$

where $x=\left[x_{1}, x_{2}, \ldots, x_{n}\right]^{T}, u=\left[u_{1}, u_{2}, \ldots, u_{n}\right]^{T}$, and $I_{n} \in$ $\mathfrak{R}^{n \times n}$. 
The following control scheme based on the predictorobserver (7) is proposed:

$$
u_{i}(t)=K_{1} \widehat{w}_{i, m}(t)+K_{2} \sum_{j=1}^{n} l_{i j}\left(\widehat{w}_{j, m}(t)-\widehat{w}_{i, m}(t)\right)
$$

for $i, j=1,2, \ldots, n, K_{1}, K_{2} \in \Re^{q \times p}$, where $l_{i j}$ are the entries of the Laplacian matrix of the graph. The control scheme (30) can be rewritten as

$$
u(t)=\left(I_{n} \otimes K_{1}-\mathscr{L} \otimes K_{2}\right) \widehat{w}_{m}(t)
$$

where $\mathscr{L} \in \mathfrak{R}^{n \times n}$ is the Laplacian of the system and $\widehat{w}_{m}=$ $\left[\widehat{w}_{1_{m}}, \ldots, \widehat{w}_{n_{m}}\right]^{T}$ is the predicted state.

The closed-loop system (29)-(31) is given by

$$
\begin{aligned}
\dot{x}(t)= & \left(I_{n} \otimes A\right) x(t) \\
& +\left(I_{n} \otimes B\right)\left(I_{n} \otimes K_{1}-\mathscr{L} \otimes K_{2}\right) \widehat{w}_{m}(t-\tau) .
\end{aligned}
$$
that

Considering that $(A \otimes B)(C \otimes D)=A C \otimes B D$, it is obtained

$$
\begin{aligned}
\dot{x}(t)= & \left(I_{n} \otimes A\right) x(t) \\
& +\left(I_{n} \otimes B K_{1}-\mathscr{L} \otimes B K_{2}\right) \widehat{w}_{m}(t-\tau) .
\end{aligned}
$$

Recalling the observation errors,

$$
\begin{aligned}
& e_{w_{1}}(t)=x(t+\bar{\tau})-\widehat{w}_{1}(t) \\
& e_{w_{f}}(t)=\widehat{w}_{f-1}(t+\bar{\tau})-\dot{\hat{w}}_{f}(t) ; \text { for } f=2,3, \ldots, m
\end{aligned}
$$

the estimated state $\widehat{w}_{m}(t-\tau)$ can be rewritten as a function of the real state and the observation errors,

$$
\widehat{w}_{m}(t-\tau)=x(t)-\sum_{f=1}^{m} e_{w_{f}}(t-f \bar{\tau})
$$

where $e_{w_{f}}=\left[e_{w 1_{f}}, \ldots, e_{w n_{f}}\right]^{T}$, producing

$$
\begin{aligned}
\dot{x}(t)= & H x(t) \\
& +\left(\mathscr{L} \otimes B K_{2}-I_{n} \otimes B K_{1}\right) \sum_{f=1}^{m} e_{w_{f}}(t-f \bar{\tau})
\end{aligned}
$$

where $H=\left(I_{n} \otimes\left(A+B K_{1}\right)-\mathscr{L} \otimes B K_{2}\right)$.

Consider now a nonsingular matrix $U \in \mathfrak{R}^{n \times n}$, such that

$$
U^{-1} \mathscr{L} U=J_{\mathscr{L}}
$$

for $J_{\mathscr{L}} \in \mathfrak{R}^{n \times n}$ representing the Jordan canonical form of the Laplacian matrix $\mathscr{L}$. able,

Considering now the globally invertible change of vari-

$$
\bar{x}=\left(U^{-1} \otimes I_{q}\right) x, \quad x=\left(U \otimes I_{q}\right) \bar{x}
$$

for $\bar{x}=\left[\bar{x}_{1}, \bar{x}_{2}, \ldots, \bar{x}_{n}\right]^{T}$; then it is possible to show that

$$
\begin{aligned}
\left(U \otimes I_{q}\right) \dot{\bar{x}}(t) \\
=\left[I_{n} \otimes\left(A+B K_{1}\right)\right]\left(U \otimes I_{q}\right) \bar{x}(t) \\
\quad-\left(\mathscr{L} \otimes B K_{2}\right)\left(U \otimes I_{q}\right) \bar{x}(t) \\
\quad+\left[\mathscr{L} \otimes B K_{2}-I_{n} \otimes B K_{1}\right] \sum_{f=1}^{m} e_{w_{f}}(t-f \bar{\tau})
\end{aligned}
$$

from where

$$
\begin{aligned}
\dot{\bar{x}}(t) & \\
= & {\left[I_{n} \otimes\left(A+B K_{1}\right)-J_{L} \otimes B K_{2}\right] \bar{x}(t) } \\
& +\left[U^{-1} \mathscr{L} \otimes B K_{2}-U^{-1} \otimes B K_{1}\right] \sum_{f=1}^{m} e_{w_{f}}(t-f \bar{\tau}) .
\end{aligned}
$$

Equation (40) can be rewritten as

$$
\dot{\bar{x}}(t)=\Gamma \bar{x}(t)+\Delta \sum_{f=1}^{m} e_{w_{f}}(t-f \bar{\tau})
$$

where

$$
\begin{aligned}
\Delta & =U^{-1} \mathscr{L} \otimes B K_{2}-U^{-1} \otimes B K_{1} \\
\Gamma & =\left[\begin{array}{ccccc}
A+B K_{1} & 0 & 0 & \cdots & 0 \\
0 & \gamma_{2} & \delta B K_{2} & \cdots & 0 \\
\vdots & \vdots & \ddots & \ddots & \vdots \\
0 & 0 & \cdots & \gamma_{n-1} & \delta B K_{2} \\
0 & 0 & \cdots & 0 & \gamma_{n}
\end{array}\right],
\end{aligned}
$$

with $\gamma_{i}=A+B K_{1}-\lambda_{L, i} B K_{2}$ for $i=2, \ldots, n$ and $\delta=0$ or $\delta=1$, depending on the structure of $J_{L}$.

Remark 12. Notice that since $H$ and $\Gamma$ are similar matrices, they have the same eigenvalues. Furthermore, in (43) it can be seen that those eigenvalues correspond to the ones of $A+B K_{1}$ and $A+B K_{1}-\lambda_{i} B K_{2}$, for $i=2,3, \ldots, n$.

\subsection{Consensus Analysis}

Lemma 13. Consider a set of $n$ agents of the form (28) with a communication topology with a directed spanning tree. Under these circumstances, solution to the consensus problem is achieved if and only if the matrices $A+B K_{1}-\lambda_{L, i} B K_{2}$, for $i=2,3, \ldots, n$, are Hurwitz.

Proof. Let the invertible matrix $P=\left[p_{1}, p_{2}, \ldots, p_{n p}\right] \in$ $\mathbb{C}^{n p \times n p}$ be such that

$$
P^{-1} H P=J_{H},
$$


with $J_{H}$ being the Jordan canonical form of $H$, defined in (36). Considering the change of coordinates,

$$
\widehat{x}=P^{-1} x, \quad x=P \widehat{x}
$$

(36) yields

$$
\begin{aligned}
\dot{\hat{x}}= & P^{-1} H P \widehat{x} \\
& +P^{-1}\left(\mathscr{L} \otimes B K_{2}-I_{n} \otimes B K_{1}\right) \sum_{f=1}^{m} e_{w_{f}}(t-f \bar{\tau}) .
\end{aligned}
$$

That is

$$
\begin{aligned}
\dot{\hat{x}}= & J_{H} \widehat{x} \\
& +P^{-1}\left(\mathscr{L} \otimes B K_{2}-I_{n} \otimes B K_{1}\right) \sum_{f=1}^{m} e_{w_{f}}(t-f \bar{\tau}) .
\end{aligned}
$$

The solution to system (47) is given by

$$
\begin{aligned}
\widehat{x}(t)= & e^{J_{H} t} \widehat{x}(0) \\
& +\sum_{f=1}^{m} \int_{0}^{t} e^{J_{H}(t-\theta)} P^{-1}\left(\mathscr{L} \otimes B K_{2}-I_{n} \otimes B K_{1}\right) \\
& \times e_{w_{f}}(\theta-f \bar{\tau}) d \theta .
\end{aligned}
$$

Since $\widehat{x}=P^{-1} x$, it is possible to write

$$
\begin{aligned}
x(t)= & P e^{J_{H} t} P^{-1} x(0) \\
& +P \sum_{f=1}^{m} \int_{0}^{t} e^{J_{H}(t-\theta)} P^{-1}\left(\mathscr{L} \otimes B K_{2}-I_{n} \otimes B K_{1}\right) \\
& \times e_{w_{f}}(\theta-f \bar{\tau}) d \theta .
\end{aligned}
$$

Let $H \in \Re^{n p \times n p}$ with eigenvectors $p_{g} \in \mathscr{C}(g=$ $1,2, \ldots, n p)$. Note, from Remark 12 , that $p_{g}(g=1,2, \ldots, n)$ correspond to the eigenvalues of $A+B K_{1}$, while eigenvectors $p_{g}(g=n+1, n+2, \ldots, n p)$ correspond to the eigenvalues of $A+B K_{1}-\lambda_{L, i} B K_{2}, i=2,3, \ldots, n$. Then, two consensus subspaces can be established, subspace $\mathscr{C}(H)$ spanned by $p_{g}(g=$ $1,2, \ldots, n)$ and a complementary consensus subspace $\overline{\mathscr{C}}(H)$ spanned by $p_{g}(g=n+1, n+2, \ldots, n p)$. Therefore, the consensus space $\mathscr{C}^{n p}$ is given by

$$
\mathscr{C}^{n p}=\mathscr{C} \oplus \overline{\mathscr{C}}
$$

Since the eigenvalues of $H$ correspond to the eigenvalues of $A+B K_{1}$ and $A+B K_{1}-\lambda_{L, i} B K_{2}, i=2,3, \ldots, n$, matrix $e^{J_{H} t}$ can be written as

$$
e^{J_{H} t}=\left[\begin{array}{cc}
e^{J_{C} t} & 0 \\
0 & e^{J_{\bar{C}} t}
\end{array}\right]
$$

defining

$$
x(0)=x_{C}(0)+x_{\bar{C}}(0)
$$

Equation (49) can now be rewritten as

$$
\begin{aligned}
x(t)= & P\left[\begin{array}{cc}
e^{J_{C} t} & 0 \\
0 & e^{J_{\bar{C}} t}
\end{array}\right] P^{-1} x(0) \\
& +P \sum_{f=1}^{m} \int_{0}^{t} e^{J_{H}(t-\theta)} P^{-1}\left(\mathscr{L} \otimes B K_{2}-I_{n} \otimes B K_{1}\right) \\
& \times e_{w_{f}}(\theta-f \bar{\tau}) d \theta .
\end{aligned}
$$

Considering that the solution of the closed-loop system (53) can be expressed as

$$
\begin{aligned}
x(t)= & x_{C}(t)+x_{\bar{C}}(t) \\
& +P \sum_{f=1}^{m} \int_{0}^{t} e^{J_{H}(t-\theta)} P^{-1}\left(\mathscr{L} \otimes B K_{2}-I_{n} \otimes B K_{1}\right) \\
& \times e_{w_{f}}(\theta-f \bar{\tau}) d \theta
\end{aligned}
$$

and noting that the limit when $t \longrightarrow \infty$ of the third term in (54) is a constant value, thus

$$
\lim _{t \longrightarrow \infty} e_{w_{f}}(t)=0 .
$$

The system consensus state can be established by means of the analysis of $x_{C}(t)$ and $x_{\bar{C}}(t)$. From (53) and (54), $x_{C}(t)$ is given by

$$
x_{C}(t)=P\left[\begin{array}{cc}
e^{J_{C} t} & 0 \\
0 & e^{J_{\bar{C}} t}
\end{array}\right] P^{-1} x_{C}(0) .
$$

Describing the initial state as

$$
\begin{aligned}
x(0)= & x_{C}(0)+x_{\bar{C}}(0) \\
= & \sum_{g=1}^{p} \alpha_{g}(0) p_{g}+\sum_{g=p+1}^{n p} \alpha_{g}(0) p_{g} \\
= & P\left[\alpha_{1}(0), \ldots, \alpha_{p}(0), 0, \ldots, 0\right]^{T} \\
& +P\left[0, \ldots, 0, \alpha_{p+1}(0), \ldots, \alpha_{n p}(0)\right]^{T}
\end{aligned}
$$

thus

$$
\begin{aligned}
& x_{C}(0)=P\left[\alpha_{1}(0), \ldots, \alpha_{p}(0), 0, \ldots, 0\right]^{T} \\
& x_{\bar{C}}(0)=P\left[0, \ldots, 0, \alpha_{p+1}(0), \ldots, \alpha_{n p}(0)\right]^{T}
\end{aligned}
$$

where $\alpha_{g}(0), g=1,2, \ldots, n p$ are real constants. From (58), $x_{C}(t)$ yields

$$
x_{C}(t)=P\left[\begin{array}{cc}
e^{J_{C} t} & 0 \\
0 & e^{J_{\bar{C}} t}
\end{array}\right]\left[\alpha_{1}(0), \ldots, \alpha_{p}(0), 0, \ldots, 0\right]^{T} .
$$

That is

$$
x_{C}(t)=P\left[\begin{array}{c}
e^{J_{C} t}\left[\alpha_{1}(0), \ldots, \alpha_{p}(0)\right]^{T} \\
0
\end{array}\right]
$$


Since $J_{C}$ is the Jordan block of $H$ corresponding to the eigenvalues of $A+B K_{1}$ and considering that the pair $(A, B)$ is controllable, it can be concluded that the pair $\left(A+B K_{1}, B\right)$ is also controllable and therefore the right choice of matrix $K_{1}$ guarantees that $x_{C}(t)$ achieves consensus. Now, $x_{\bar{c}}(t)$ is given by

$$
\begin{aligned}
& x_{\overline{\mathrm{C}}}(t) \\
& \quad=P\left[\begin{array}{cc}
e^{J_{\mathrm{C}} t} & 0 \\
0 & e^{J_{\bar{C}} t}
\end{array}\right]\left[0, \ldots, 0, \alpha_{p+1}(0), \ldots, \alpha_{n p}(0)\right]^{T}
\end{aligned}
$$

that yields

$$
x_{\bar{C}}(t)=P\left[\begin{array}{c}
0 \\
e^{J \bar{C}^{t}}\left[\alpha_{p+1}(0), \ldots, \alpha_{n p}(0)\right]^{T}
\end{array}\right] .
$$

And since $J_{\overline{\mathrm{C}}}$ is the Jordan block of $H$ corresponding to the eigenvalues of $A+B K_{1}-\lambda_{L, i} B K_{2}$, for $i=2,3, \ldots, n$, then it can be concluded that for system (28) to achieve consensus matrices $A+B K_{1}-\lambda_{L, i} B K_{2}, i=2,3, \ldots, n$, must be Hurwitz, which implies

$$
\lim _{t \longrightarrow \infty} x_{\bar{C}}(t)=0 .
$$

Considering (60) and (63), the system consensus value is given by

$$
\begin{aligned}
& \lim _{t \rightarrow \infty} x(t)=\lim _{t \rightarrow \infty}\left\{P\left[\begin{array}{c}
\left.e^{J_{C} t}\left[\alpha_{1}(0), \ldots, \alpha_{p}(0)\right]^{T}\right] \\
0
\end{array}\right]\right. \\
& +P \sum_{f=1}^{m} \int_{0}^{t} e^{J_{H}(t-\theta)} P^{-1} \\
& \left.\quad \times\left(\mathscr{L} \otimes B K_{2}-I_{n} \otimes B K_{1}\right) e_{w_{f}}(\theta-f \bar{\tau}) d \theta\right\} .
\end{aligned}
$$

Since it has been shown that $\lim _{t \rightarrow \infty} e_{w_{f}}(t)=0$,

$$
\begin{aligned}
\lim _{t \rightarrow \infty} P & \sum_{f=1}^{m} \int_{0}^{t} e^{J_{H}(t-\theta)} P^{-1}\left(\mathscr{L} \otimes B K_{2}-I_{n} \otimes B K_{1}\right) \\
& \times e_{w_{f}}(\theta-f \bar{\tau}) d \theta=E
\end{aligned}
$$

with a real constant vector $E$; then,

$$
\lim _{t \rightarrow \infty} x(t)=\lim _{t \rightarrow \infty} P\left[\begin{array}{c}
e^{J_{C} t}\left[\alpha_{1}(0), \ldots, \alpha_{p}(0)\right]^{T} \\
0
\end{array}\right]+E .
$$

Finally, defining

$$
\left[\beta_{1}(t), \ldots, \beta_{p}(t)\right]^{T}=e^{J_{\mathrm{C}} t}\left[\alpha_{1}(0), \ldots, \alpha_{p}(0)\right]^{T},
$$

and considering that Lemma 4 from [7] proves that the first $p$ eigenvectors of $H$ are given by $p_{g}=\mathbf{1}_{n} \otimes c_{j}$ for $g=1, \ldots, p$

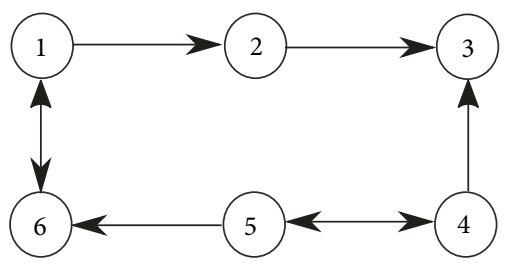

FIGURE 1: Communication topology.

where $c_{g}$ are the eigenvectors of $A+B K_{1}$ and $\mathbf{1}_{n} \in \Re^{n}$ is the vector with all of its entries equal to one, the consensus value (66) yields

$$
\begin{aligned}
\lim _{t \rightarrow \infty} x(t)= & \lim _{t \rightarrow \infty} \mathbf{1}_{n} \\
& \otimes\left[c_{1}, \ldots, c_{p}\right]\left[\beta_{1}(t), \ldots, \beta_{p}(t)\right]^{T}+E .
\end{aligned}
$$

Remark 14. It should be noted that the previous proof is based on the corresponding proof presented in [7] with the difference that the proof presented in this work includes the predicted state used in the consensus protocol which results in a consensus value affected by the initial injection errors, represented by $E$ in (68).

Remark 15. Notice that the prediction-observation scheme proposed here can compensate the time-delay of the agents when it is placed either at the input or at the output. In the case of agents with input time-delay, each agent will estimate its own future state and send it to its neighbors which will use them to calculate its own control, thus compensating their input time-delay. In the case where the agents present an output time-delay, once again, each agent would estimate its current state from its delayed output and send the undelayed estimated state to the other agents. In both cases, the consensus will be achieved by means of a decentralized algorithm.

\section{Numerical Evaluations}

Numerical evaluations of the proposed solution carried out consider two cases, the Multiagent Supporting System (MASS) composed by six agents presented in [7] and a multiagent system formed by six agents with unstable dynamics whose stabilization is achieved in [31]. For simplicity, in both cases the communication topology is used, depicted in Figure 1, with the corresponding Laplacian matrix given by

$$
\mathscr{L}=\left[\begin{array}{cccccc}
1 & 0 & 0 & 0 & 0 & -1 \\
-1 & 1 & 0 & 0 & 0 & 0 \\
0 & -1 & 2 & -1 & 0 & 0 \\
0 & 0 & 0 & 1 & -1 & 0 \\
0 & 0 & 0 & -1 & 1 & 0 \\
-1 & 0 & 0 & 0 & -1 & 2
\end{array}\right] .
$$




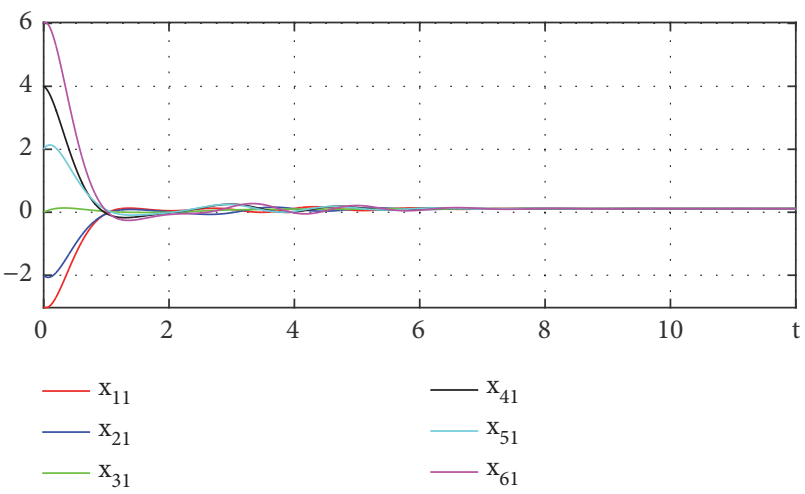

FIgURE 2: Time evolution of the state, MASS system. $\tau=1.2 \mathrm{~s}$.

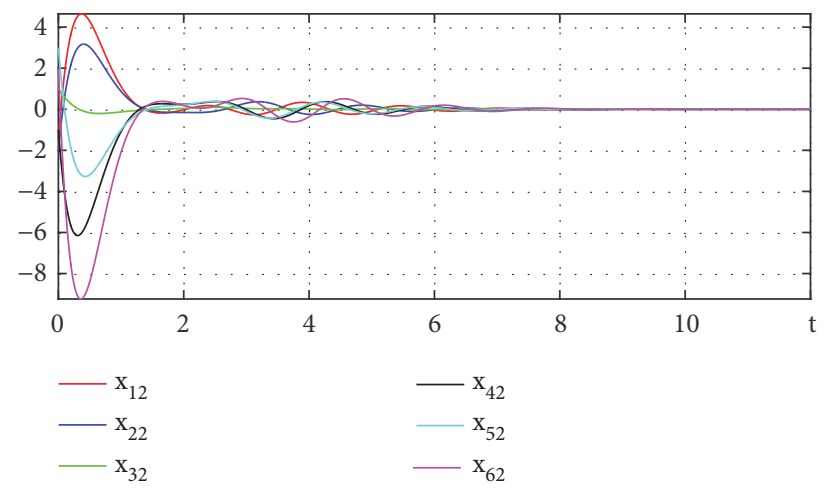

FIGURE 3: Time evolution of the state, MASS system. $\tau=1.2 s$.

Example 1. In the case of the MASS system, the dynamics of each agent is given by

$$
\dot{x}_{i}(t)=\left[\begin{array}{cc}
0 & 1 \\
\frac{-k}{m_{i}} & \frac{-D}{m_{i}}
\end{array}\right] x_{i}(t)+\left[\begin{array}{l}
0 \\
1
\end{array}\right] u\left(t-\tau_{i}\right),
$$

where $x_{i}(t)=\left[z_{i}(t), \dot{z}_{i}(t)\right]^{T}, z_{i}(t)$ is the height of each agent, $m_{i}$ is the mass, $D$ is the damping at each agent, $k$ is the stiffness, and $u_{i}(t)$ is the control input. The implementation of the predictor considers a partition of $m=4$ and the system parameters $m_{i}=10.6, k=120$, and $D=50.5$. The initial conditions are set as

$$
x_{0}=[-3,-1.5,-2,-2,0,1,4,-1,2,3,6,2]^{T} .
$$

The gain matrix for the predictor-observer is given by $\mathrm{E}_{i}=[1,0 ; 4,0]^{T}$ and the consensus gain vectors $K_{1}=$ $[11.3208,-0.2358]$ and $K_{2}=[8.8687,1]$. The delay considered is $\tau=1.2 s$; the results are given in Figures 2, 3, 4, and 5 that represent the evolution in time of the state, observation errors, and control signal, respectively. The evolution of the logarithm of the homogeneous norm of the prediction error is depicted in Figure 6 to show its exponential convergence. It can be seen that with the partition of the predictor-observer large delays can be compensated.
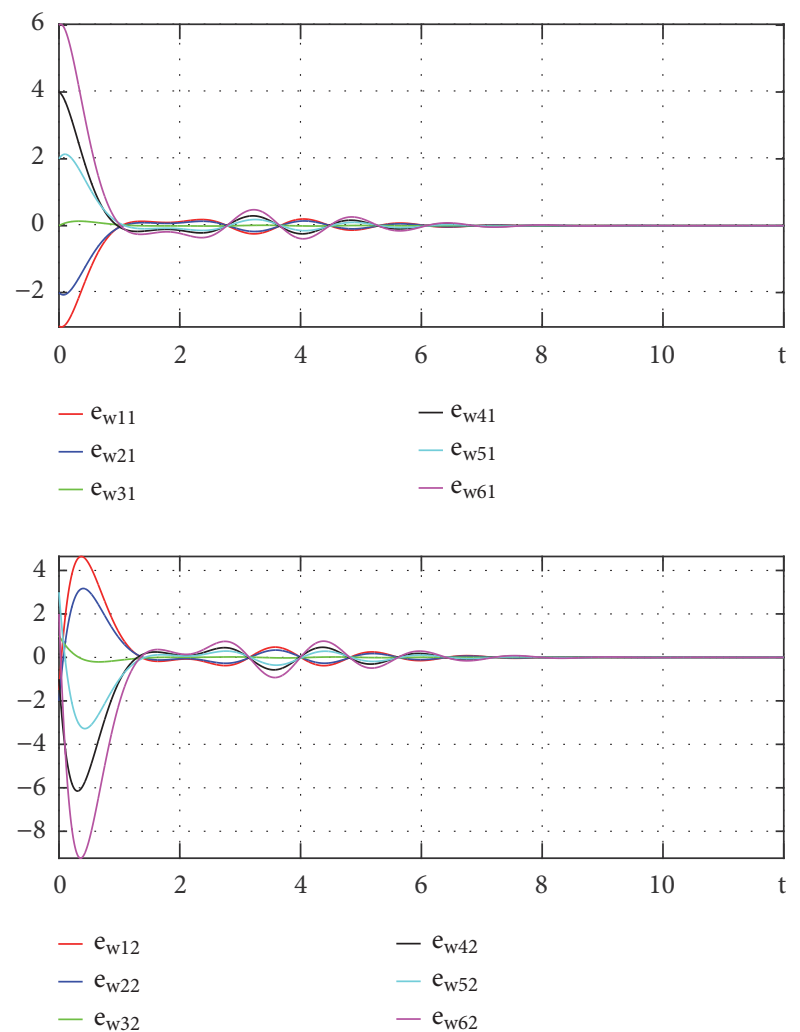

FIGURE 4: Time evolution of the estimation errors, MASS system. $\tau=1.2 s$.

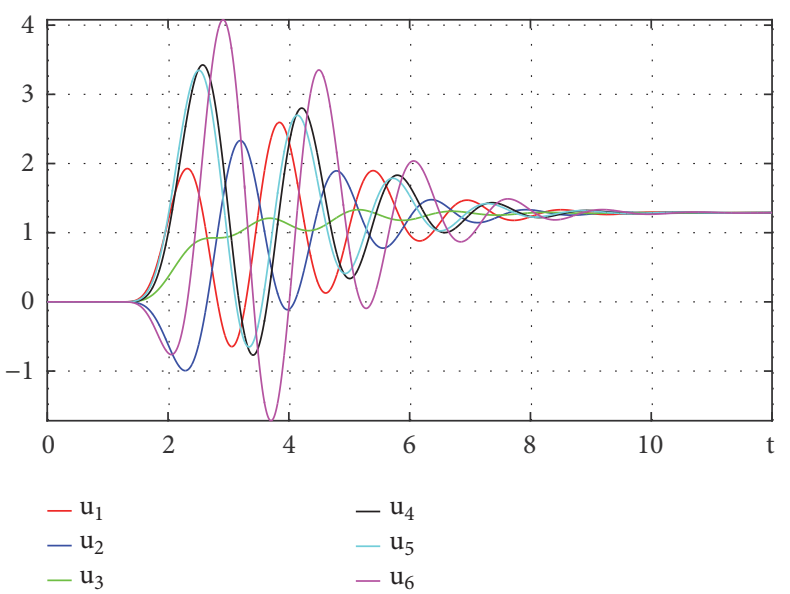

FIGURE 5: Time evolution of the control signal, MASS system. $\tau=$ $1.2 s$.

Example 2. In a more challenging situation, an unstable system presented in [31] is taken into account to form a multiagent system with $n=6$ and the communication topology presented in Figure 1. The dynamics of each agent is given by

$$
\begin{aligned}
& \dot{x}(t)=\left[\begin{array}{cc}
-5 & -6 \\
3 & 4
\end{array}\right] x(t)+\left[\begin{array}{l}
0 \\
4
\end{array}\right] u(t-\tau) \\
& y(t)=\left[\begin{array}{ll}
-2 & 2
\end{array}\right] x(t) .
\end{aligned}
$$




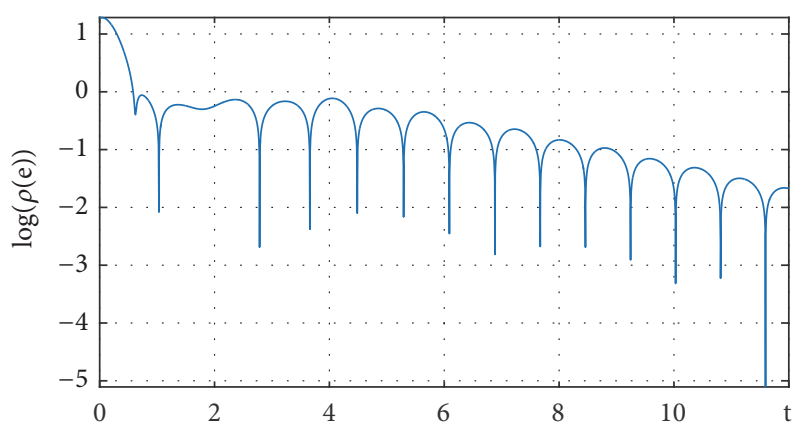

Figure 6: Time evolution of the logarithm of the prediction error, MASS system. $\tau=1.2 \mathrm{~s}$.
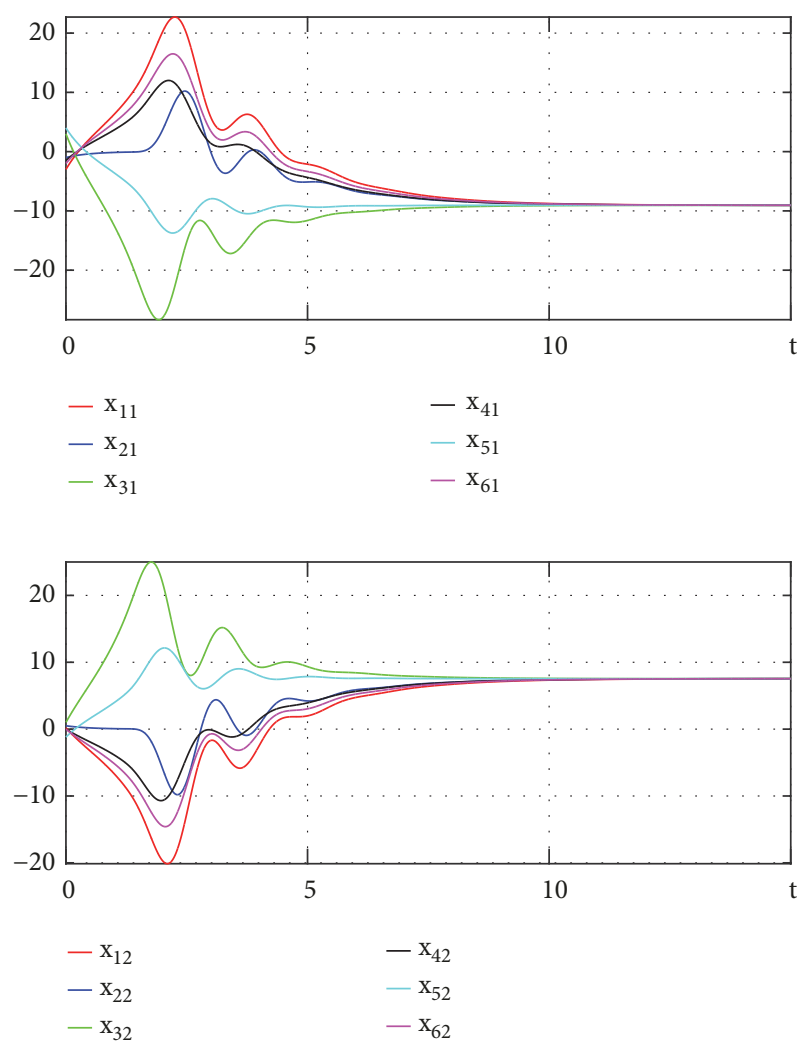

Figure 7: Time evolution of the state, unstable agents. $\tau=1 \mathrm{~s}$.

The delay of the system is $\tau=1 \mathrm{~s}$, the partition of the observer is $m=4$, and the initial conditions are set as

$$
x_{0}=[-3,0.2,-1,0.5,3,1,-1.5,0,4,-1.2,-2,0]^{T} .
$$

The gain of each subpredictor is given by $L_{i}=\left[\begin{array}{lll}-0.8212 & 0.7263\end{array}\right]^{T}$, and the control gains are $K_{1}=\left[\begin{array}{ll}-0.5417 & -0.75\end{array}\right]$ and $K_{2}=\left[\begin{array}{ll}-4 & 0.3\end{array}\right]$. Figures 7 and 8 present the evolution in time of the state of the system and the prediction errors, respectively, where after a transient period the desired convergence is obtained; Figure 9 presents the evolution of the control signal. The logarithm of the norm of the prediction error is presented in Figure 10.
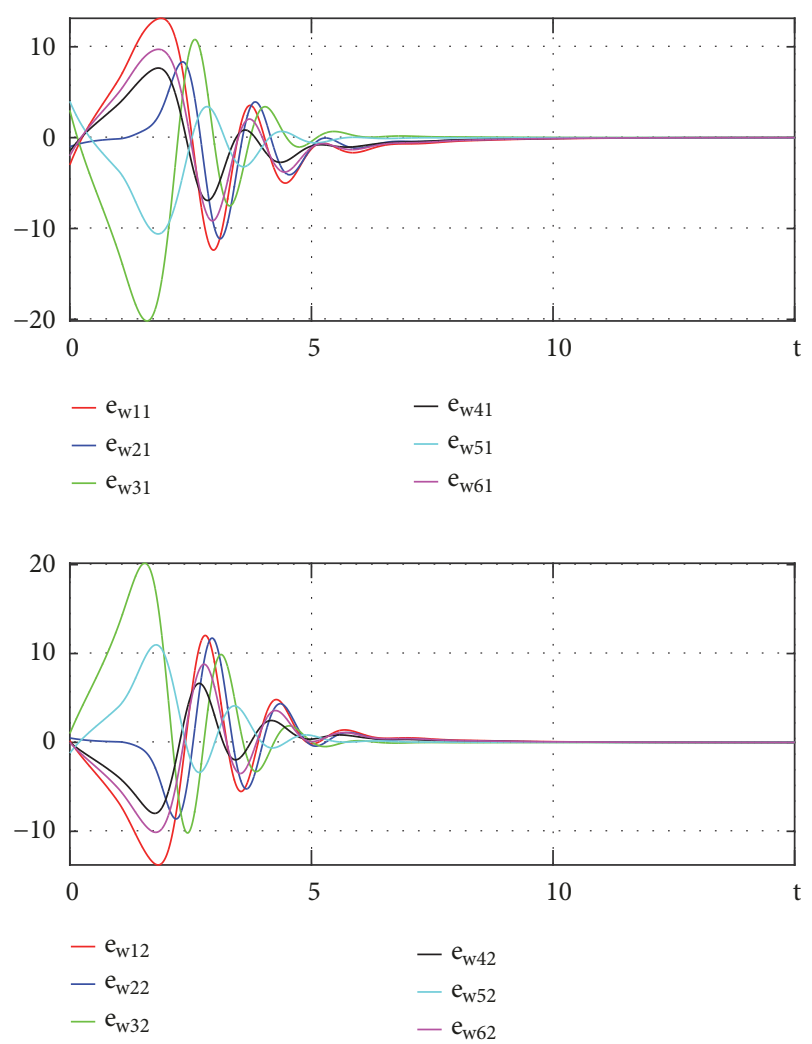

FIGURE 8: Time evolution of the estimation errors, unstable agents. $\tau=1 s$.

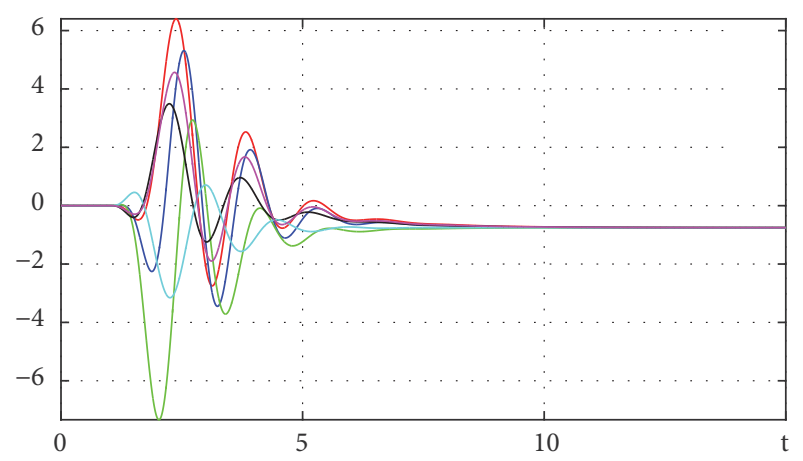

$$
\begin{array}{ll}
-\mathrm{u}_{1} & -\mathrm{u}_{4} \\
-\mathrm{u}_{2} & -\mathrm{u}_{5} \\
-\mathrm{u}_{3} & -\mathrm{u}_{6}
\end{array}
$$

FIGURE 9: Time evolution of the control signal, unstable agents. $\tau=$ $1 s$.

\section{Conclusions}

This work deals with the consensus problem for multiagent systems with LTI dynamics and input time-delay. A modified partitioned predictor-observer is proposed to estimate the future state values of the agents. These predicted states are used in the consensus protocol in order to compensate the input time-delays. Formal proofs for the convergence of the predictor and consensus are given. The performed numerical evaluations show that a larger number of partitions 


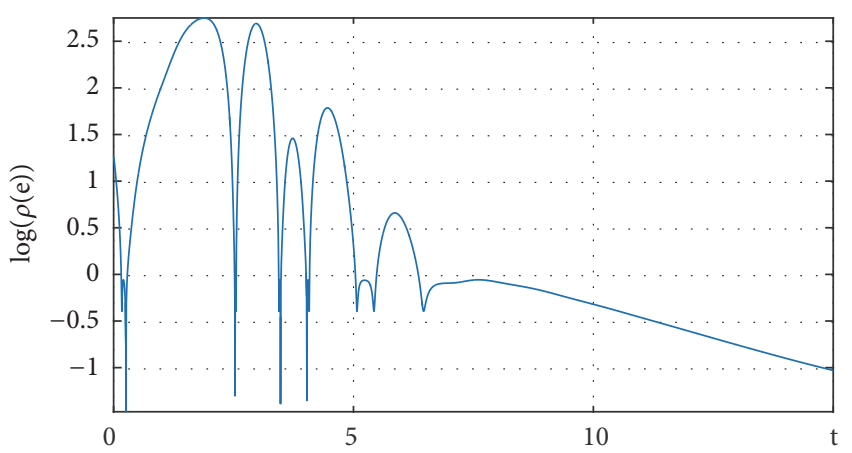

FIGURE 10: Time evolution of the logarithm of the prediction error, unstable agents. $\tau=1 s$.

in the predictor-observer results in an improvement of the response of the system and in a larger time-delay that can be compensated.

\section{Data Availability}

No data were used to support this study.

\section{Conflicts of Interest}

The authors declare that they have no conflicts of interest.

\section{References}

[1] W. Ren and E. Atkins, "Distributed multi-vehicle coordinated control via local information exchange," International Journal of Robust and Nonlinear Control, vol. 17, no. 10-11, pp. 1002-1033, 2007.

[2] R. Olfati-Saber and R. M. Murray, "Distributed cooperative control of multiple vehicle formations using structural potential functions," IFAC World Congress, 2002.

[3] J. A. Fax and R. M. Murray, "Information flow and cooperative control of vehicle formations," IEEE Transactions on Automatic Control, vol. 49, no. 9, pp. 1465-1476, 2004.

[4] R. Olfati-Saber and R. M. Murray, "Consensus problems in networks of agents with switching topology and time-delays," IEEE Transactions on Automatic Control, vol. 49, no. 9, pp. 15201533, 2004.

[5] A. Jadbabaie, J. Lin, and A. S. Morse, "Coordination of groups of mobile autonomous agents using nearest neighbour rules," IEEE Transactions on Automatic Control, vol. 48, no. 6, pp. 988-1001, 2003.

[6] L. Moreau, "Consensus and cooperation in networked multiagent systems," IEEE Transactions on Automatic Control, vol. 50, no. 2, pp. 169-182, 2005.

[7] J. Xi, N. Cai, and Y. Zhong, "Consensus problems for high-order linear time-invariant swarm systems," Physica A: Statistical Mechanics and its Applications, vol. 389, no. 24, pp. 5619-5627, 2010.

[8] W. Ren and R. W. Beard, Distributed Consensus in Multi-Vehicle Cooperative Control. Theory and Applications, Springer-Verlag, London, UK, 2008.

[9] L. Peng, J. Yingmin, D. Junping, and Y. Shiying, "Distributed consensus protocol for second-order agents with fixed topology and time-delay," in Proceedings of the 26th Chinese Control Conference (CCC '07), pp. 577-581, 2007.

[10] Z. Yan, D. Wu, and Y. Liu, "Consensus of discrete multiagent system with various time delays and environmental disturbances," Entropy, vol. 16, no. 12, pp. 6524-6538, 2014.

[11] D. Lee and M. W. Spong, "Agreement with non-uniform information delays," in Proceedings of the American Control Conference, IEEE, 2006.

[12] P. Lin and Y. Jia, "Consensus of a class of second-order multiagent systems with time-delay and jointly-connected topologies," IEEE Transactions on Automatic Control, vol. 55, no. 3, pp. 778-784, 2010.

[13] P. Lin and Y. Jia, "Consensus of second-order discrete-time multi-agent systems with nonuniform time-delays and dynamically changing topologies," Automatica, vol. 49, no. 5, pp. 21542158, 2009.

[14] J. Xi, Z. Shi, and Y. Zhong, "Consensus analysis and design for high-order linear swarm systems with time-varying delays," Physica A: Statistical Mechanics and its Applications, vol. 390, no. 23-24, pp. 4114-4123, 2011.

[15] J. Xi, Z. Wang, G. Liu, and Y. Zhong, "Consensus problems for linear time-varying swarm systems with topology variances and multiple time delays," in Proceedings of the 2013 32nd Chinese Control Conference (CCC '13), pp. 192-198, 2013.

[16] X. Luo, X. Li, S. Li, and X. Guan, "Consensus for linear multiagent systems with constant and time-varying communication delays via delay-decomposition approach," in Proceedings of the 11th World Congress on Intelligent Control and Automation, pp. 353-358, 2014.

[17] Y. Cao, T. Oguchi, P. Verhoeckx, and H. Nijmeijer, "Consensus control for a multiagent system with time delays," Mathematical Problems in Engineering, vol. 2017, Article ID 4063184, 11 pages, 2017.

[18] S. Mehra and S. R. Sahoo, "Trajectory tracking with input delay in multi-agent system: Double integrator case," in Proceedings of the 2016 International Conference on Unmanned Aircraft Systems (ICUAS '16), pp. 387-393, 2016.

[19] S. Mehra, A. Sen, and S. R. Sahoo, "Trajectory tracking in heterogeneous multi-agent system without and with input delay," in Proceedings of the 2017 Indian Control Conference (ICC '17), pp. 338-343, 2017.

[20] B. Zhou and Z. Lin, "Consensus of high-order multi-agent systems with large input and communication delays," Automatica, vol. 50, no. 2, pp. 452-464, 2014.

[21] C. Wang, Z. Zuo, Z. Lin, and Z. Ding, "A truncated prediction approach to consensus control of lipschitz nonlinear multiagent systems with input delay," IEEE Transactions on Control of Network Systems, vol. 4, no. 4, pp. 716-724, 2017.

[22] P. Shi and Q. K. Shen, "Observer-based leader-following consensus of uncertain nonlinear multi-agent systems," International Journal of Robust and Nonlinear Control, 2017.

[23] O. J. M. Smith, "Closer control of loops with deadtime," Chemical Engineering Progress, vol. 53, no. 5, pp. 217-219, 1957.

[24] K. Watanabe and M. Ito, "A process-model control for linear systems with delay," IEEE Transactions on Automatic Control, vol. 26, no. 6, pp. 1261-1269, 1981.

[25] W. Michiels and D. Roose, "Time-delay compensation in unstable plants using delayed state feedback," in Proceedings of the 40th IEEE Conference on Decision and Control, '01, vol. 2, pp. 1433-1437, IEEE, 2001. 
[26] A. Manitius and A. W. Olbrot, "Finite spectrum assignment problem for systems with delays," IEEE Transactions on Automatic Control, vol. 24, no. 4, pp. 541-553, 1979.

[27] Z. Artstein, "Linear systems with delayed controls: a reduction," IEEE Transactions on Automatic Control, vol. 27, no. 4, pp. 869879, 1982.

[28] L. Mirkin and N. Raskin, "Every stabilizing dead-time controller has an observer-predictor-based structure," Automatica, vol. 39, no. 10, pp. 1747-1754, 2003.

[29] S. Mondié and W. Michiels, "Finite spectrum assignment of unstable time-delay systems with a safe implementation," IEEE Transactions on Automatic Control, vol. 48, no. 12, pp. 22072212, 2003.

[30] B. Zhou, "Input delay compensation of linear systems with both state and input delays by nested prediction," Automatica, vol. 50, no. 5, pp. 1434-1443, 2014.

[31] M. Najafi, S. Hosseinnia, F. Sheikholeslam, and M. Karimadini, "Closed-loop control of dead time systems via sequential subpredictors," International Journal of Control, vol. 86, no. 4, pp. 599-609, 2013.

[32] F. Sheikholeslam, S. Hosseinnia, M. Najafi, and Q. Wang, "Robust $\mathrm{H} \infty$ control of single input-delay systems based on sequential sub-predictors," IET Control Theory \& Applications, vol. 8, no. 13, pp. 1175-1184, 2014.

[33] F. Cacace, F. Conte, A. Germani, and G. Palombo, "Optimal control of linear systems with large and variable input delays," Systems \& Control Letters, vol. 89, pp. 1-7, 2016.

[34] F. Cacace, A. Germani, and C. Manes, "Predictor-based control of linear systems with large and variable measurement delays," International Journal of Control, vol. 87, no. 4, pp. 704-714, 2014.

[35] F. Mazenc and M. Malisoff, "Stabilization and robustness analysis for time-varying systems with time-varying delays using a sequential subpredictors approach," Automatica, vol. 82, pp. 118-127, 2017.

[36] A. Germani, C. Manes, and P. Pepe, "A new approach to state observation of nonlinear systems with delayed output," Institute of Electrical and Electronics Engineers Transactions on Automatic Control, vol. 47, no. 1, pp. 96-101, 2002.

[37] M. Velasco-Villa, J. Heras-Godínez, J. A. Vázquez-Santacruz, and V. Fragoso-Rubio, "Delayed consensus problem for single and double integrator systems," Mathematical Problems in Engineering, vol. 2015, Article ID 461098, 15 pages, 2015.

[38] V. Fragoso-Rubio, M. Velasco-Villa, and M. A. HernándezPérez, "Consensus problem for linear time invariant systems with time-delay," in Proceedings of the National Conference of Automatic Control '17, pp. 90-95, 2017.

[39] C. Godsil and G. Royle, Algebraic Graph Theory, Springer, New York, NY, USA, 2001.

[40] W. Ren, R. W. Beard, and E. M. Atkins, "Information consensus in multivehicle cooperative control," IEEE Control Systems Magazine, vol. 27, no. 2, pp. 71-82, 2007.

[41] W. Michiels, K. Engelborghs, P. Vansevenant, and D. Roose, "Continuous pole placement for delay equations," Automatica, vol. 38, no. 5, pp. 747-761, 2002.

[42] K. Gu, V. L. Kharitonov, and J. Chen, Stability of Time-Delay Systems, Birkhauser, 2003.

[43] R. E. Bellman and K. L. Cooke, Differential-Difference Equations, Rand Corporation, 1963.

[44] I. Estrada-Sánchez, M. Velasco-Villa, and H. Rodríguez-Cortés, "Prediction-based control for nonlinear systems with input delay," Mathematical Problems in Engineering, vol. 2017, Article ID 7415418, 11 pages, 2017. 


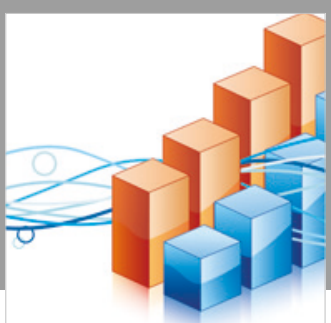

Advances in

Operations Research

\section{-n-m}
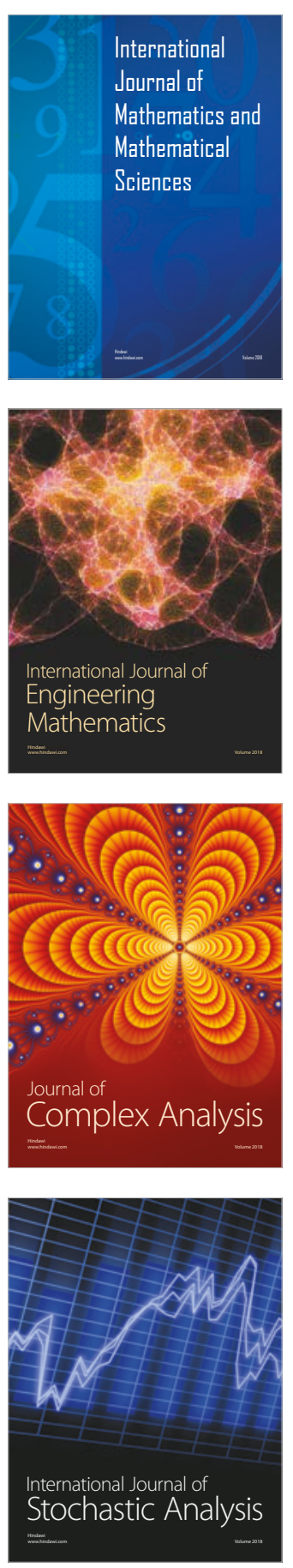
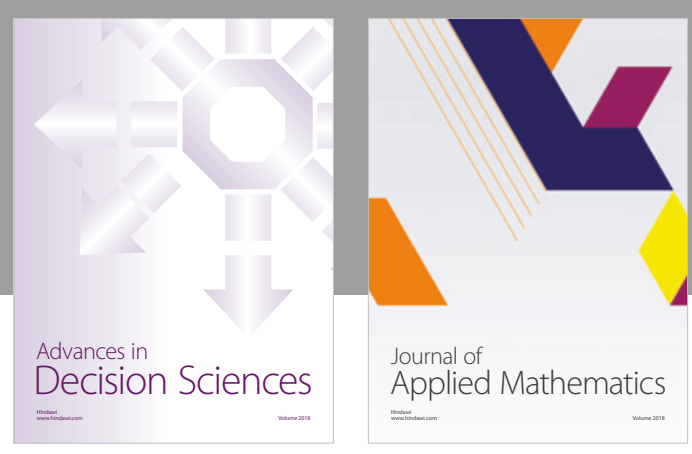

Journal of

Applied Mathematics
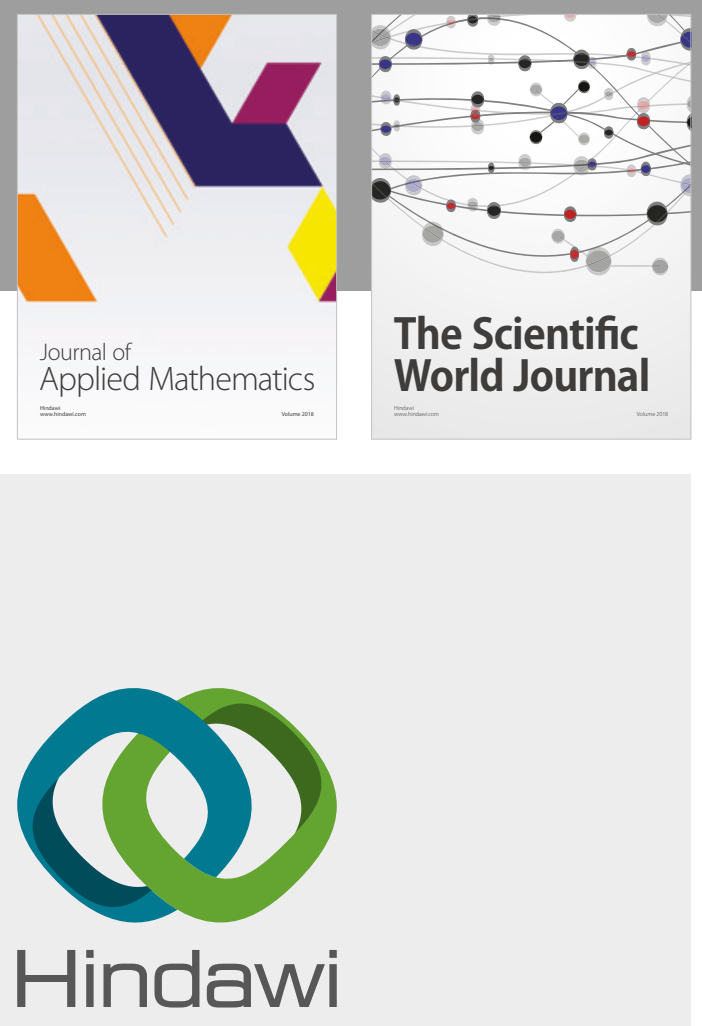

Submit your manuscripts at

www.hindawi.com

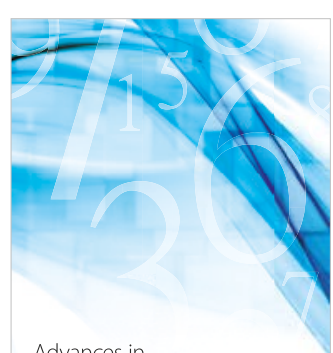

Advances in
Numerical Analysis
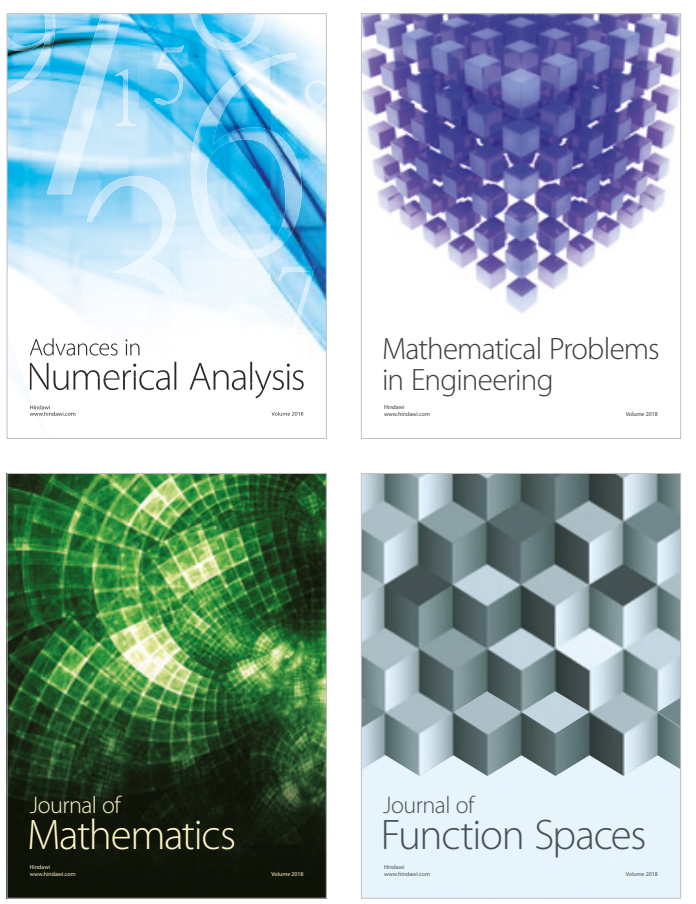

Mathematical Problems in Engineering

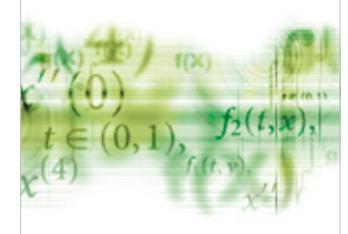

International Journal of

Differential Equations

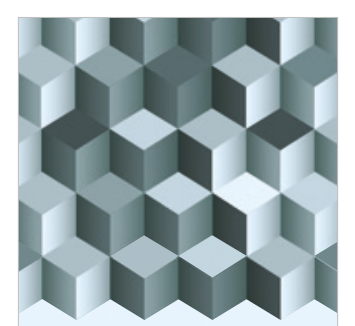

Journal of

Function Spaces
The Scientific

World Journal

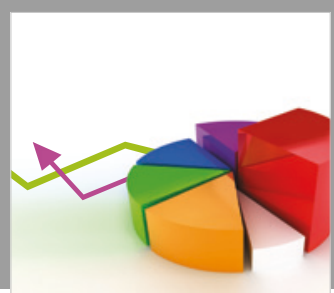

Journal of

Probability and Statistics
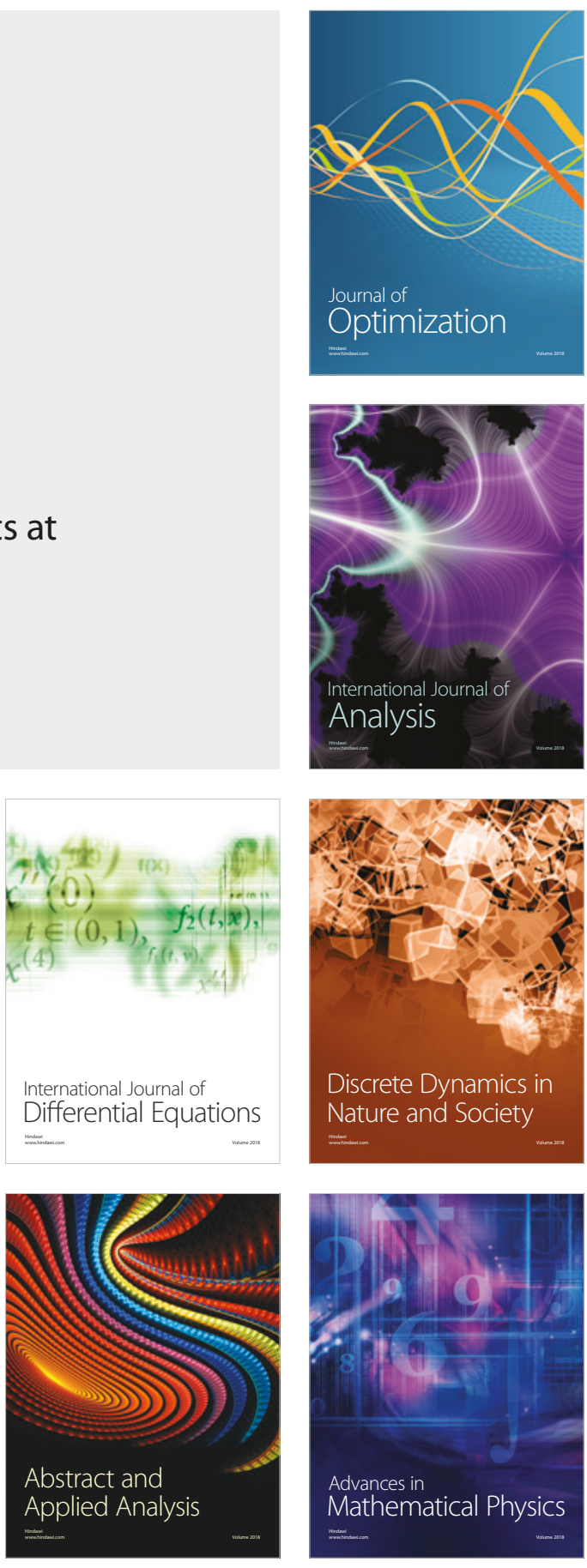